\title{
Honoring the 65th Birthday of Professor Barry M. Trost
}

It is a pleasure to write an appreciation of the research of Barry M. Trost and in particular to acknowledge his many contributions to heterocyclic chemistry.

Barry received his BA from the University of Pennsylvania in '62 and his Ph.D. from M.I.T. in '65 working with H.O. House on "The Structure and Reactivity of Enolate Ions". He then went directly to the University of Wisconsin where he rose through the various professorial ranks to Professor in '69. He chaired the Chemistry Department from '80-82' and was Vilas Professor when he left Wisconsin for Stanford in '87. He chaired the Chemistry Department at Stanford from '96-'02 and now is their Tamaki Professor of Humanities and Sciences.

Barry is interested in developing new synthetic reactions and reagents, primarily for use in synthesizing complex molecules from readily available and simple starting materials. He likes to use transition metals to carry out chemo-, regio-, diastereo- and especially enantioselective reactions. A favorite approach is to severely limit the use of unnecessary reaction and purification steps, as favored in his "green" chemistry. Metal-catalyzed cycloaddition is a common topic, especially to make in this non-traditional way rings that are 5-, 7-, 9- membered and larger, including heterocyclic rings. Palladium is of great interest together with a considerable portion of the Periodic Chart including nickel, chromium, molybdenum, ruthenium, iron and tungsten. Bimetallic complexes composed of two different metals are especially prominent. Barry and his group like to make sulfur compounds act either as nucleophiles or electrophiles, a nice trick. His target compounds include $\beta$-lactam antibiotics, ionophores, and steroids in a list that just seems to go on-and-on.

Barry M. Trost has been much honored previously and the record is too long to include here. He started as an A.P. Sloan Fellow in '67-'69 and has been continuously recognized from that time. He has been awarded by his peers numerous ACS honors, including the Arthur C. Cope Award in '04. He is a member of the National Academy of Sciences and holds numerous honorary doctorates. His teaching skills were recognized in '93 with the Bing Teacher Award.

Barry contributes to our profession by acting on various editorial boards. He served as associate editor of JACS from '74-'80 and continues to act as editor of CHEMTRACTS-Organic Chemistry to this day.

More than 720 publications bear his name as well as 14 patents. ISI lists him as a member of the World's 50 Most Cited Chemists. Plenary lectures at national and international conferences number more than 220. 
However, the dedication of this issue of Heterocycles is somewhat unique. It is perhaps the first time that Barry has been honored specifically for his accomplishments in heterocyclic chemistry. Heterocyclic chemistry is not emphasized in American academic chemical circles and within memory one of our practitioners wrote that no self-respecting American organic chemist would ever describe himself (yes a little chauvinistic) as a heterocyclic chemist! Yet, among the many compounds that the Trost research group has synthesize is a large collection of heterocyclic molecules, ranging from aziridines to the heretofore little known 5H-oxazol-4-ones to nucleosides to oxindoles and chromans, some using his novel DYKAT (dynamic kinetic asymmetric transformation) process.

His usual focus is on the economy of synthetic steps and controlled stereochemistry, often making use of his favorite metal catalysts to achieve these ends.

Barry Trost has contributed significantly to modern developments in heterocyclic chemistry and it's a real pleasure to recognize him in Heterocycles. We wish him many more productive years!

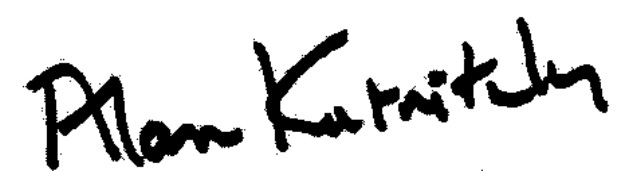

Alan R. Katritzky

Gainesville, Florida, USA 